The Official Journal of the Kettil Bruun Society for Social and Epidemiological Research on Alcohol

\title{
Risk Factors for Fracture in Alcohol-dependent, Pre-menopausal Females
}

\author{
Nicholas R. Fuggle ${ }^{1}$, Joseph Singer ${ }^{2}$, Michael A. Clynes ${ }^{1}$, Beth Curtis ${ }^{1}$, Pallavi Wyawahare ${ }^{2}$, Hayley J. Denison ${ }^{2}$, \\ Moira Gilmour $^{3}$, Geoff Robinson ${ }^{3}$, and Elaine M. Dennison ${ }^{1,2}$ \\ ${ }^{1}$ MRC Lifecourse Epidemiology Unit, University of Southampton, Southampton, United Kingdom \\ ${ }^{2}$ School of Biological Sciences, Victoria University of Wellington, Wellington, New Zealand \\ ${ }^{3}$ Capital and Coast District Health Board and Medical Research Institute of New Zealand, Wellington, New Zealand
}

\begin{abstract}
Aims: Alcoholism is known to be associated with increased risk of fracture. This study aimed to study bone turnover following alcohol detoxification and to investigate lifestyle factors for low bone density that might coexist with alcohol dependency, which might be amenable to modification.

Method: Pre-menopausal female participants were recruited from an alcohol-use dependency unit to a cross-sectional study. A lifestyle questionnaire, including alcohol history, smoking, physical activity, dietary calcium intake, falls, and fracture history was completed. Quantitative heel ultrasonography was performed and broadband ultrasound attenuation (BUA), speed of sound (SOS), $t$ score, and $z$ score were recorded. Blood was taken for bone-turnover markers at baseline and day 5 following admission for alcohol withdrawal.
\end{abstract}

Results: The mean age (SD) of alcohol dependent participants was 41.6 (8.3) years, with participants reporting high levels of current cigarette smoking, physical inactivity, and falls. BUA, SOS, $t$ scores, and $z$ scores were lower than the age-matched reference range in alcohol-dependent participants. Levels of type 1 procollagen (P1NP) increased significantly after five days $(p<$ $.001)$.

Conclusions: Alcohol-dependent, pre-menopausal individuals have multiple risk factors for fracture, beyond alcohol excess. These should be addressed and targeted as modification may reduce fracture risk, especially given the apparent recovery of bone turnover on the withdrawal of alcohol.

Alcohol is consumed by $80 \%$ of the adult population in New Zealand (Ministry of Health, 2015) with an estimated annual social cost of NZ\$4.4 billion (Slack, Nana, Webster, Stokes, $\&$ Wu, 2009). Research by the Health Promotion Agency has shown that the amount of alcohol consumed by women is increasing (Huckle, Yeh, Lin, \& Jensen, 2013). The relationship between alcohol consumption and fractures is complex; a meta-analysis demonstrated an increased risk of fracture with high alcohol consumption, but lower levels of alcohol consumption appeared beneficial to bone health (Berg et al., 2008). Negative effects of high amounts of alcohol on bone may reflect a direct effect due to alcohol or other constituents of alcoholic beverages (such as silicon and polyphones), endocrine disturbance, or disorder of calcium metabolism. The increased risk of fracture may also be related to coexisting lifestyle factors, such as cigarette smoking, low levels of physical activity, or poor diet.
The various constituents of alcoholic beverages interact with bone differently. These include ethanol, which may have a possible dose-dependent toxic effect on osteoblasts (AlvisaNegrin et al., 2009); silicon, which is positively associated with bone mineral density (BMD) in cortical bone (Jugdaohsingh et al., 2004); and polyphones (found in beer and red wine), which manifest osteogenic effects through estrogen receptors (Powell et al., 2005). Markers of bone formation, such as osteocalcin, have been suppressed by high alcohol intake, and there are data suggesting that this suppression is reversible following abstinence (Malik, Gasser, Moncayo, Kemmler, \& Wolfgang Fleischhacker, 2012). Endocrinopathies can develop as a result of alcohol dependence, including Cushings (Smalls, Kloppenborg, Njo, Knoben, \& Ruland, 1976), hypogonadism (Diamond et al., 1990), and secondary amenorrhea (Hay, 2003), all of which are associated with secondary osteoporosis. Vitamin D levels appear to be lower in individuals consuming high

Correspondence: Professor Elaine Dennison, Victoria University of Wellington, Kelburn Parade, Wellington 6140, New Zealand. Telephone: +64 4-472- 1000, Email: elaine.dennison@vuw.ac.nz

Financial support: Wellington Medical Research Foundation

Declaration of interest: The authors have no conflict of interest to declare.

Keywords: alcohol, fracture, osteoporosis, pre-menopausal, female 
levels of alcohol. This may result in subsequent poor calcium absorption and raised parathyroid hormone measurements, which may lead to reduced bone mineral density (Laitinen, Välimäki, \& Keto, 1991). The effects of alcohol on bone health can be assessed by measurement of blood levels of bone-turnover markers, as bone is in a constant state of remodeling, which in health is demonstrated as balanced bone resorption and formation.

While many studies have considered these direct consequences of alcoholism on bone health (Peris et al., 1994; Lindholm et al., 1991), far fewer have considered whether other lifestyle factors detrimental to bone health might coexist in individuals with alcohol dependence. Such information would be helpful when counseling patients attending alcohol detoxification programs. These inpatient stays represent a window of opportunity when the patients is motivated to effect lifestyle change and when assessment of lifestyle factors other than alcohol intake can be discussed.

Many lifestyle factors are known to be associated with poor acquisition of peak bone mass (Weaver et al., 2016) and accelerated bone loss in later life (Dennison \& Cooper, 2007). Factors that are commonly reviewed and reported as important include physical activity, dietary calcium intake, and cigarette smoking. Peak bone mass is attained by approximately thirty years of age; in their review, Weaver and colleagues (2016) highlighted the importance of diet, especially calcium intake and vitamin $\mathrm{D}$, and reported on associations between physical activity levels and bone mass. A systematic review has highlighted the detrimental effects of smoking on bone health (Kanis et al., 2005), and other studies have considered how clustering of risk factors might impact bone health (Zhang et al., 2016).

Many studies have been performed in men with alcohol dependency (Hyeon et al., 2008; Spencer, Rubio, Rubio, Indreika, \& Seitam, 1986), but far fewer data are available in women. There are conflicting data regarding the role of alcohol on bone health in pre-menopausal females. In one study of 892 adolescent females, an overall negative association of alcohol intake with BMD was observed (Lucas, Fraga, Ramos, \& Barros, 2012); however, in another similar cohort of 202 adolescents, no such association was found (Dorn, 2013).

This study aimed to measure bone health and bone turnover and relate these to additional lifestyle risk factors for fracture in a group of alcohol-dependent, pre-menopausal women. Our hypothesis was that women who were alcohol dependent would have other lifestyle risk factors for poor bone health, such as cigarette smoking, low dietary calcium intake, and low levels of weight-bearing physical activity. If alcohol detoxification is successful, these additional risk factors would also need to be addressed to improve bone health. We hypothesized that changes in bone turnover would be apparent within the admission detoxification period and might provide positive feedback to women to motivate them to make lifestyle changes.

\section{Method}

\section{Study Design}

The study was designed as an observational cross-sectional study in the locality of Wellington, New Zealand. Ethical approval for this research was granted by the Health and Disability Ethics Committee (HDEC), reference NTY/12/04/024/AM06, and conducted in accordance with the Declaration of Helsinki general principles for medical research. There were two components: a survey with a questionnaire, adapted from one used extensively in previous epidemiological studies of lifestyle risk factors for poor bone health (Syddall et al., 2005), and a laboratory component where blood tests were performed and assays of bone turnover measured on days 1 and 5 .

\section{Study Setting}

The study was sited in the Medical Detoxification Unit of Kenepuru Hospital in Porirua, New Zealand.

\section{Selection Criteria}

Premenopausal females aged 22-55 years were recruited at the start of their alcohol-use detoxification admission. All women gave informed consent and were in the hospital for a minimum of five days. Exclusion criteria were age outside this range; males; inability to give informed consent.

\section{Data Collection}

A questionnaire comprising three sections was completed, covering aspects regarding risk factors for fracture and bone health. The first section detailed alcohol consumption habits, including age drinking started, number of units consumed, and type of alcohol consumed; smoking history (as age at started smoking) and number of cigarettes smoked per day; medical history to consider secondary causes for osteoporosis; falls in the last year; personal and family history of fractures; and history of menarche, menstruation, and menopause. The second section focused on medication history and physical activity, included quantifying physical activity acutely (over the previous seven days) and in the longer term (preceding six months). Moderate physical activity was described as that which "made breathing harder than normal" and vigorous physical activity was defined as that which "made breathing a lot harder than normal." Physical-activity questions were taken from a validated questionnaire (Boon, Hamlin, Steel, \& Ross, 2010). The third section included calcium intake and diet. The questionnaire was administered by the researcher who obtained consent.

In this study we assessed bone health using quantitative heel ultrasound (HUS). This was a pragmatic decision, as there was no direct access to dual-energy X-ray absorptiometry (DXA) scanning during the inpatient stay. Following calibration and adequate positioning, HUS was performed on both heels using a GE Achilles+ EXPII Bone Ultrasonometer. The recorded measurements included broadband ultrasound attenuation (BUA), speed of sound (SOS), stiffness index, $t$ score (the number of standard deviations from a mean for a young individual of the same 
sex), and $z$ score (the number of standard deviations from a mean for an individual of the same sex and age).

Blood samples were taken on the day of admission, including serum procollagen type $1 \mathrm{~N}$ propeptide (P1NP) and serum C-terminal cross-linking telopeptide of type 1 collagen (CTX). P1NP and CTX were repeated on discharge five days later. CTX was measured using the Roche Elecsys Beta-Crosslaps method. P1NP was measured using the Roche Elecsys 2010 method (Clynes et al., 2014). Study participants' characteristics were summarized using means and standard deviations for continuous variables and numbers and percentages for binary and categorical variables. Normality was assessed and, due to bone-turnover markers not being normally distributed, the Wilcoxon signed-rank test was used to investigate the change in these parameters over time. We performed a power calculation prior to undertaking this study. Assuming a mean (SD) CTX of $0.14(0.072)$ micrograms/L among premenopausal women, a study of 42 premenopausal women has $90 \%$ power at the 5\% significance level to detect a change in CTX of $25 \%$ over the follow-up period. Assuming a mean (SD) P1NP of 23.08 (9.52) micrograms/L among premenopausal women, a study of 42 premenopausal women has $88 \%$ power at the $5 \%$ significance level to detect a change in P1NP of $20 \%$ over the follow-up period

\section{Results}

We recruited 53 patients from the alcohol dependence unit (demographics shown in Table 1).

\section{Table 1}

\section{Demographics of participants}

\begin{tabular}{lc}
\hline Demographic parameter & Mean (SD) \\
\hline Age (years) & $41.6(8.3)$ \\
BMI $\left(\mathrm{kg} / \mathrm{m}^{2}\right)$ & $26.2(6.4)$ \\
Calcium intake (mg/day) & $445.1(321.7)$ \\
Smoking & $37(69.8)$ \\
$\quad$ Number of current smokers (\%) & $17.5(17.9)$ \\
Pack years per smoker [mean(SD)] & \\
Alcohol & $17.9(12.9)$ \\
$\quad$ Units of alcohol consumption per day & $24.2(8.5)$ \\
$\quad$ Years of alcohol consumption & \\
$\quad$ [mean(SD)] & \\
Physical Activity & $19(35.8)$ \\
$\quad$ Number inactive (\%) & $11(20.8)$ \\
$\quad$ Number moderately active (\%) & $23(43.3)$ \\
$\quad$ Number active (\%) & \\
Falls & $39(73.6)$ \\
$\quad$ Number who had fallen in last year (\%) & \\
Fractures & $27(50.9)$ \\
$\quad$ Number sustaining fracture as adult $(\%)$ & $6(11.3)$ \\
Family history of hip fracture (\%) &
\end{tabular}

The mean age (SD) for alcohol-dependent participants was 41.6 (8.3) years. The mean body mass index (BMI) (SD) for participants was $26.2(6.4) \mathrm{kg} / \mathrm{m}^{2}$. The mean calcium intake for participants was $445.1 \mathrm{mg} /$ day $(\mathrm{SD}=321.7 \mathrm{mg} /$ day $)$. In terms of tobacco history, $37(69.8 \%)$ of the participants currently smoked with a mean of $17.5(\mathrm{SD}=17.9)$ pack years per smoker. Participants consumed a mean of 17.9 (SD $=12.9$ ) units per day, had commenced consumption of alcohol at a mean age of $17.4(\mathrm{SD}=7.1)$ years, and had consumed alcohol for a mean of $24.2(\mathrm{SD}=8.5)$ years. In terms of physical activity level, 19 (35.8\%) were inactive, 11 (20.8\%) were moderately active, and $23(43.3 \%)$ were active. Falls occurred commonly, with 39 (73.6\%) having fallen in the last year, and $27(50.9 \%)$ had sustained a fracture as an adult. Six $(11.3 \%)$ had a family history of hip fracture.

We calculated the number of modifiable risk factors for each participant of cigarette smoking, physical inactivity, and dietary calcium intake below the recommended daily intake of $800 \mathrm{mg}$ /day. Of our sample, 23 women $(43.4 \%)$ had one risk factor, 18 women (34\%) had two risk factors, and 12 $(22.6 \%)$ women had 3 risk factors. All participants had at least one lifestyle risk factor that might be addressed to improve bone health following discharge.

Bone ultrasonometer results included BUA measures with a mean (SD) of $105.2 \mathrm{~dB} / \mathrm{MHz}(15.7 \mathrm{~dB} / \mathrm{MHz})$ and SOS with a mean (SD) of $1547.3 \mathrm{~m} / \mathrm{s}(37.2 \mathrm{~m} / \mathrm{s}) . T$ scores and $z$ scores were low, with means (SD) of -1.2 (1.4) and -0.6 (1.3) respectively. Mean level of $25-\mathrm{OH}$ vitamin D was 54.0 $\mathrm{nmol} / \mathrm{L}$ (SD $24.9 \mathrm{nmol} / \mathrm{L})$.

P1NP and CTX levels were measured at baseline and day 5 as biomarkers of bone turnover. Numbers of participants having blood testing were 31 on day 1 , and, on day 5,30 for CTX and 31 for P1NP. Levels of P1NP, a marker of bone formation, rose significantly from baseline to day 5 ( $p<$ $.001)$. Change in levels of CTX, a marker of bone resorption, did not reach statistical significance $(p=.61)$.

Table 2

Prevalence of risk factors for bone health in study
sample compared with national data, Australia and New
Zealand

\begin{tabular}{lcc}
\cline { 2 - 3 } & $\begin{array}{c}\text { Study } \\
\text { sample }\end{array}$ & $\begin{array}{c}\text { Australia \& } \\
\text { New Zealand }\end{array}$ \\
\hline $\begin{array}{l}\text { Dietary calcium intake below } \\
800 \mathrm{mg} / \text { day }\end{array}$ & $74 \%$ & $25 \%$ \\
$\begin{array}{l}\text { Physical activity insufficient } \\
\text { for health benefits }\end{array}$ & $36 \%$ & $45 \%$ \\
Current smoking rate & $69 \%$ & $12 \%$ \\
\hline
\end{tabular}

\section{Discussion}

We performed an observational study to investigate the bone-health parameters and risk factors for fracture in a group of pre-menopausal female patients admitted to an alcohol-dependency unit. We undertook this work to highlight other factors besides alcohol abuse that coexist in this population that might be addressed during a hospital admission, when motivation to effect lifestyle change is high and there is access to dietetic and other support services. We found that these pre-menopausal, alcohol-dependent individuals reported several lifestyle risk factors for fracture 
other than alcohol dependence, including current cigarette smoking, physical inactivity, and low dietary calcium intake. Women reported high rates of prior fracture and a high incidence of falls. Parameters of bone health measured by HUS, including BUA, SOS, $t$ score, and $z$ score, showed below-average values. Interestingly, there was a significant increase in P1NP, representing recovery in bone formation, from day 1 to day 5 of detoxification, with no significant change in CTX levels over the period of study. These results suggest the potential for recovery of bone health following alcohol detoxification and warrants further study in larger samples.

These findings also emphasize the importance of assessing and addressing coexisting risk factors for fracture beyond alcohol in this group of females. Indeed, at the time of detoxification, individuals have made an active decision to engage with healthcare and are therefore ripe for education regarding other measures that could be taken to improve bone health and reduce fracture risk. The results also suggest that detoxification can lead to a significant improvement in bone turnover over a relatively short period of time (in this case, 5 days), highlighting the importance of abstaining from alcohol in order to improve bone health. It is worth considering that the intervention of alcohol detoxification goes beyond alcohol withdrawal to include vitamin supplementation and nutritional improvement for inpatients. This highlights the importance of holistic approach to the management of alcohol detoxification.

Few studies have considered the role of lifestyle factors that might influence bone mass and may coexist alongside alcohol dependency. In one study, Knudsen et al. highlighted the high prevalence of nutritional deficiencies in alcoholdependent adults (2014). Others have suggested that multiple risk factors may be an issue in individuals with this condition (Drake et al., 2012), but in general this has not been addressed. That osteoporosis may coexist with alcohol dependency in men is well established (Martin-Gonzalez et al., 2017), but far fewer data are available in women, although one study did highlight the high prevalence of vertebral fractures in this group (Haramati, Alterman, Israel, Mallavurapu, \& Haramati, 1998).

At the time of writing, this is the first study characterizing the bone health of a population of pre-menopausal females recruited from an alcohol-dependency unit. This is particularly important as most previous pre-menopausal studies have been performed on adolescent females (Lucas et al., 2012; Dorn et al., 2013). Although this specific population has not been previously studied, our findings regarding bone structure (as measured by HUS) concur with previous data in other groups. In an Australian study, a randomly selected population of 534 men aged 65 years or older were subject to DXA and quantitative ultrasound and subanalyzed for alcohol intake via a food frequency questionnaire. Alcohol consumption was found to be inversely associated with BMD and quantitative ultrasound measures (Coulson et al., 2013). In a Japanese study of 256 pre-menopausal and 585 post-menopausal women, a positive trend was found for the effect on alcohol on mean bone stiffness (calculated using BUA and SOS readings from quantitative ultrasonography) in the post-menopausal group. Interestingly, no effect was observed in the premenopausal population ( $p=.66$ ) (Fukuharu et al., 2001). This seems to point against our results; however, it should be noted that of the 256 pre-menopausal females, only 44 met this criterion, and alcohol intake in this study was defined at a cut-off of consumption on three or more days per week. The alcohol group in this study therefore differed considerably to our participant group.

In terms of bone markers, high levels of alcohol consumption are typically associated with reduced bone mineral density and likely reduction in bone turnover. Interestingly, our serum marker findings suggest that, as participants abstained, levels of P1NP were significantly less suppressed and bone formation recovered as a result. Although our study did not demonstrate a significant change in CTX, other studies have shown changes in osteocalcin (Malik et al., 2012). Two studies have considered bone turnover in men following alcohol withdrawal. In both these studies, bone formation was significantly reduced compared to controls, with a trend towards normalization 10 days after alcohol withdrawal.

The mean level of 25-OH vitamin D in this population was higher than expected, given previous findings of low levels (Lindholm et al., 1991). This may reflect seasonal sunlight exposure; although there was wide variation in levels, our results suggest that the role played by vitamin D deficiency in the bone mineral density of these participants is likely to be minimal compared to other parameters. Our subjects, who have significant alcohol dependency, reported frequent falls. This fact, in consort with lower measures of bone health and additional risk factors for fracture, places this group at an increased risk of fracture and thus morbidity and mortality.

This study has a number of limitations, including a relatively small sample size for an observational study. The nature of the population studied meant that compliance was a significant issue and, though the aim was to perform repeated phlebotomy at greater time intervals following detoxification to measure bone turnover markers, this was not possible due to poor rates of follow-up after discharge from hospital. DXA would have allowed measurement of bone density; however, this was not available in the study location, and it would not have been appropriate to transfer participants to another unit for the purposes of this study.

In addition, a significant limitation of our study is the lack of a control group against which to compare national lifestyle characteristics. However, some comparisons against Australian and New Zealand populations are possible. Specifically, using Australian Household Survey data, in $2014-15,80.6 \%$ of Australians aged 18 years and older had consumed alcohol in the past year. In the same year, $31.7 \%$ of Australian women aged 18 years and older reported consuming more than four standard drinks at least once in the past year, similar to 2011-12 (44.7\%). Young adults were more likely to report alcohol intake at this level than other ages. In $2014-15,60.6 \%$ of females aged $18-24$ years consumed more than four standard drinks at least once in the past year. In 2014-15, in the same survey, $12.1 \%$ of women 
smoked daily, while $55.5 \%$ of $18-64$-year-olds participated in sufficient physical activity in the last week (more than 150 minutes of moderate physical activity or more than 75 minutes of vigorous physical activity, or an equivalent combination of both, including walking). In a New Zealand study (Horwath, Parnell, Wilson, \& Russell, 2001), 20\% of New Zealanders and one in four women had intakes below the UK Estimated Average Requirements for calcium. Milk and milk products were the major sources of the nation's calcium intake.

In conclusion, our findings demonstrate that in a group of pre-menopausal women admitted to an alcohol-dependency unit, there were a number of risk factors for fracture. These extended beyond simple alcohol excess to include cigarette smoking, low dietary calcium intake, previous fracture, and high incidence of falling. These risk factors should all be targeted in assessment of bone health, as modification may decrease risk of fracture. Abstention from alcohol led to a significant increase in levels of P1NP at day 5 of detoxification, suggesting an acute improvement in bone formation. Further research is required to elucidate the longer-term effects on bone turnover following detoxification.

To our knowledge, this is the first study that has considered the prevalence of risk factors for osteoporosis that coexist with alcohol dependency. Our results suggest that many women has several risk factors that need to be considered alongside alcohol withdrawal to improve bone health.

\section{Acknowledgements}

This study was supported by a grant from the Wellington Medical Research Foundation. We thank the staff and patients of Kenepuru Alcohol Dependency Unit who supported this study.

\section{References}

Alvisa-Negrín, J., González-Reimers, E., SantolariaFernández, F., García-Valdecasas-Campelo, E., Valls, M. R. A., Pelazas-González, R., . . . \& de los Ángeles Gómez-Rodríguez, M. (2009). Osteopenia in alcoholics: Effect of alcohol abstinence. Alcohol \& Alcoholism, 44(5), 468-475.

Berg, K. M., Kunins, H. V., Jackson, J. L., Nahvi, S., Chaudhry, A., Harris Jr, K. A., . . . \& Arnsten, J. H. (2008). Association between alcohol consumption and both osteoporotic fracture and bone density. The American Journal of Medicine, 121(5), 406-418.

Boon, R. M., Hamlin, M. J., Steel, G. D., \& Ross, J. J. (2010). Validation of the New Zealand Physical Activity Questionnaire (NZPAQ-LF) and the International Physical Activity Questionnaire (IPAQLF) with accelerometry. British Journal of Sports Medicine, 44(10), 741-746.

Clynes, M., Wyawahare, P., Robinson, G., Denison, H., Evans, G., Gilmour, M., \& Dennison, E. (2014). Bone health in female alcoholics. Osteoporosis International, 25, S706-S707.

Coulson, C. E., Williams, L. J., Brennan, S. L., Berk, M., Kotowicz, M. A., Lubman, D. I., \& Pasco, J. A. (2013). Alcohol consumption and body composition in a population-based sample of elderly Australian men. Aging Clinical and Experimental Research, 25(2), 183-192.

Dennison, E., \& Cooper, C. (2007). Lifestyle and constitutional risk factors. In S. Lanham-New, T. O'Neill, R. Morris, D. Skelton, \& A. Sutcliffe [Eds.], Managing Osteoporosis (pp. 69-80). Oxfordshire, England: Atlas Medical Publishing.

Diamond, T. H., Stiel, D., Lunzer, M., Wilkinson, M., Roche, J., \& Posen, S. (1990). Osteoporosis and skeletal fractures in chronic liver disease. Gut, 31(1), 82-87.

Dorn, L. D., Beal, S. J., Kalkwarf, H. J., Pabst, S., Noll, J. G., \& Susman, E. J. (2013). Longitudinal impact of substance use and depressive symptoms on bone accrual among girls aged 11-19 years. Journal of Adolescent Health, 52(4), 393-399.

Drake, M. T., Murad, M. H., Mauck, K. F., Lane, M. A., Undavalli, C., Elraiyah, T., . . \& \& Hazem, A. (2012). Risk factors for low bone mass-related fractures in men: A systematic review and meta-analysis. The Journal of Clinical Endocrinology \& Metabolism, 97(6), 1861-1870.

Fukuharu, M., Sato, J., Ohsawa, I., Oshida, Y., Kuriki, K., Shibata, K., . . \& \& Sato, Y. (2001). Effects of lifestyle factors on ultrasonographically determined bone health in Japanese women. Public Health, 115(2), 146-151.

Haramati, L. B., Alterman, D. D., Israel, G. M., Mallavurapu, R., \& Haramati, N. (1998). Complications related to osteopenia in the thoracic spine on admission chest radiographs of substance abuse detoxification patients. Skeletal Radiology, 27(10), 565-568.

Hay, J. E. (2003). Osteoporosis in liver diseases and after liver transplantation. Journal of Hepatology, 38(6), 856-865.

Horwath, C., Parnell, W. R., Wilson, N. C., \& Russell, D. G. (2001). Attaining optimal bone status: Lessons from the 1997 National Nutrition Survey. New Zealand Medical Journal, 114(1128), 138.

Huckle, T., Yeh, L. C., Lin, J., \& Jensen, V. G. L. (2013). Trends in alcohol consumption and alcohol-related harms among females in New Zealand. Wellington, New Zealand: Health Promotion Agency.

Hyeon, J. H., Gwak, J. S., Hong, S. W., Kwon, H., Oh, S. W., \& Lee, C. M. (2016). Relationship between bone mineral density and alcohol consumption in Korean men: The fourth Korea National Health and Nutrition Examination Survey (KNHANES), 2008-2009. Asia Pacific Journal of Clinical Nutrition, 25(2), 308-315.

Jugdaohsingh, R., Tucker, K. L., Qiao, N., Cupples, L. A., Kiel, D. P., \& Powell, J. J. (2004). Dietary silicon intake is positively associated with bone mineral density in men and premenopausal women of the Framingham Offspring Cohort. Journal of Bone and Mineral Research, 19(2), 297-307. 
Kanis, J. A., Johnell, O., Odén, A., Johansson, H., De Laet, C., Eisman, J. A., . . \& \& Melton, L. J. (2005). Smoking and fracture risk: A meta-analysis. Osteoporosis International, 16(2), 155-162.

Knudsen, A. W., Jensen, J. E. B., Nordgaard-Lassen, I., Almdal, T., Kondrup, J., \& Becker, U. (2014). Nutritional intake and status in persons with alcohol dependency: Data From an outpatient treatment programme. European Journal of Nutrition, 53(7), 1483-1492.

Laitinen, K., Välimäki, M., \& Keto, P. (1991). Bone mineral density measured by dual-energy X-ray absorptiometry in healthy Finnish women. Calcified Tissue International, 48(4), 224-231.

Lindholm, J., Steiniche, T., Ramussen, E., Thamsborg, G., Nielsen, I. O., Brockstedt-Ramussen, H. E. L. L. E., . . . \& Schou, C. (1991). Bone disorder In men with chronic alcoholism: A reversible disease? The Journal of Clinical Endocrinology \& Metabolism, 73(1), 118 124.

Lucas, R., Fraga, S., Ramos, E., \& Barros, H. (2012). Early initiation of smoking and alcohol drinking as a predictor of lower forearm bone mineral density in late adolescence: A cohort study in girls. PLoS One, 7(10), e46940.

Malik, P., Gasser, R. W., Moncayo, R., Kemmler, G., \& Wolfgang Fleischhacker, W. (2012). Markers of bone resorption and formation during abstinence in male alcoholic patients. Alcoholism: Clinical and Experimental Research, 36(12), 2059-2064.

Martín-González, C., González-Reimers, E., Quintero-Platt, G., Cabrera-García, P., Romero-Acevedo, L., GómezRodríguez, M. Á., . . . \& Santolaria-Fernández, F. (2017). Lipid profile and bone mineral density in heavy alcoholics. Clinical Nutrition. S0261-5614(17)313687.

Ministry of Health. (2015). Annual update of key results 2014/15: New Zealand Health Survey. Wellington, New Zealand: Ministry of Health.

Nyquist, F., Ljunghall, S., Berglund, M., \& Obrant, K. (1996). Biochemical markers of bone metabolism after short and long time ethanol withdrawal in alcoholics. Bone, 19(1), 51-54.

Pepersack, T., Fuss, M., Otero, J., Bergmann, P., Valsamis, J., \& Corvilain, J. (1992). Longitudinal study of bone metabolism after ethanol withdrawal in alcoholic patients. Journal of Bone and Mineral Research, 7(4), 383-387.

Peris, P., Parés, A., Guan̄abens, N., Del Río, L., Pons, F., De Osaba, M. J. M., . . \& \& Muñoz-Gómez, J. (1994). Bone mass improves in alcoholics after 2 years of abstinence. Journal of Bone and Mineral Research, 9(10), 1607-1612.

Powell, J. J., McNaughton, S. A., Jugdaohsingh, R., Anderson, S. H. C., Dear, J., Khot, F., . . . \& BoltonSmith, C. (2005). A provisional database for the silicon content of foods in the United Kingdom. British Journal of Nutrition, 94(5), 804-812.

Slack, A., Nana, G., Webster, M., Stokes, F., \& Wu, J. (2009). Costs of harmful alcohol and drug use. Wellington, New Zealand: Business and Economic Research Limited.
Smalls, A. G., Kloppenborg, P. W., Njo, K. T., Knoben, J. M., \& Ruland, C. M. (1976). Alcohol-induced Cushingoid Syndrome. British Medical Journal, 2(6047), 1298.

Spencer, H., Rubio, N., Rubio, E., Indreika, M., \& Seitam, A. (1986). Chronic alcoholism. Frequently overlooked cause of osteoporosis in men. The American Journal of Medicine, 80(3), 393-397.

Syddall, H. E., Aihie Sayer, A., Dennison, E. M., Martin, H. J., Barker, D. J. P., \& Cooper, C. (2005). Cohort profile: The Hertfordshire Cohort Study. International Journal of Epidemiology, 34(6), 1234-1242.

Weaver, C. M., Gordon, C. M., Janz, K. F., Kalkwarf, H. J., Lappe, J. M., Lewis, R., . . \& \& Zemel, B. S. (2016). The National Osteoporosis Foundation's position statement on peak bone mass development and lifestyle factors: A systematic review and implementation recommendations. Osteoporosis International, 27(4), 1281-1386.

Zhang, J., Jameson, K., Sayer, A. A., Robinson, S., Cooper, C., \& Dennison, E. (2016). Accumulation of risk factors associated with poor bone health in older adults. Archives of Osteoporosis, 11(1), 3. 